\title{
Vein of trolard sign on noncontrast computed tomography in Behcet's disease
}

\author{
Tamra Ranasinghe ${ }^{1}$, William D. Freeman ${ }^{2}$
}

${ }^{1}$ Department of Neuroscience and ${ }^{2}$ Department of Neurology, Mayo Clinic Jacksonville, FL

A 45-year old male with Behcet's disease (BD) with prior oral aphthous and genital ulcers diagnosed outside our facility presented to the emergency department (ED) with two weeks of progressive headaches with abrupt onset dysarthria, left lower facial and left arm weakness. The patient had recently moved from another state to our region and stopped taking his warfarin for history of deep vein thrombosis, and had not established care with a rheumatologist, neurologist or primary care physician. Because of his unknown history at the local emergency room a noncontrast computed tomography (CT) head was originally interpreted as negative for acute stroke (Figure 1A). His symptoms did transiently improve in the ED and he was later discharged home. Subsequently he returned after a generalized tonic-clonic seizure, and a second noncontrast CT (Figure 1B) showed a hyperdense right cortical vein of Trolard (VOT) and delta $(\Delta)$ signs indicating saggital sinus thrombosis and a right hemisphere subcortical hemorrhage. He was transferred to our facility for a possible aneurysmal subarachnoid hemorrhage. At our facility we performed a CT venogram which confirmed the localization of the venous thrombus (Figure $1 \mathrm{C}$ and 1D). The patient was placed on IV

heparin initially, and on warfarin and clinically improved back to normal within a few days. He was discharged from our hospital with followup at our clinic with a rheumatology expert and neurology clinic. Cerebral venous thrombosis (CVT) is an important complication in $\mathrm{BD}(1,2)$ which may result in significant morbidity or mortality. In this patient, with history of BD with headache, a high degree of clinical suspicion should be raised for CVT. Therefore, the initial noncontrast CT head should be examined closely for abnormalities, and in this case was found to be consistent with CVT. We propose the "VOT sign" as a new, highly specific finding on noncontrast head CT suggestive of CVT which should prompt consideration of a CT venogram to confirm the diagnosis. The "VOT sign" is an useful sign even in patients with unknown history because it is highly abnormal and to our knowledge it has not been described before. Noncontrast CT head may be falsely negative in 10-20\% of cases with proven CVT (3) compared to CT or MR venography. Mimics of a positive hyperdense sagittal sinus include causes of elevated hematocrit such as polycythemia, dehydration, or CVT. Management of CVT is initial anticoagulation with IV heparin followed by vitamin $\mathrm{K}$ antagonists such as warfarin. Intracranial hemorrhage that occurs as a consequence of CVT is not a contraindication for anticoagulation (3), although this remains controversial. 

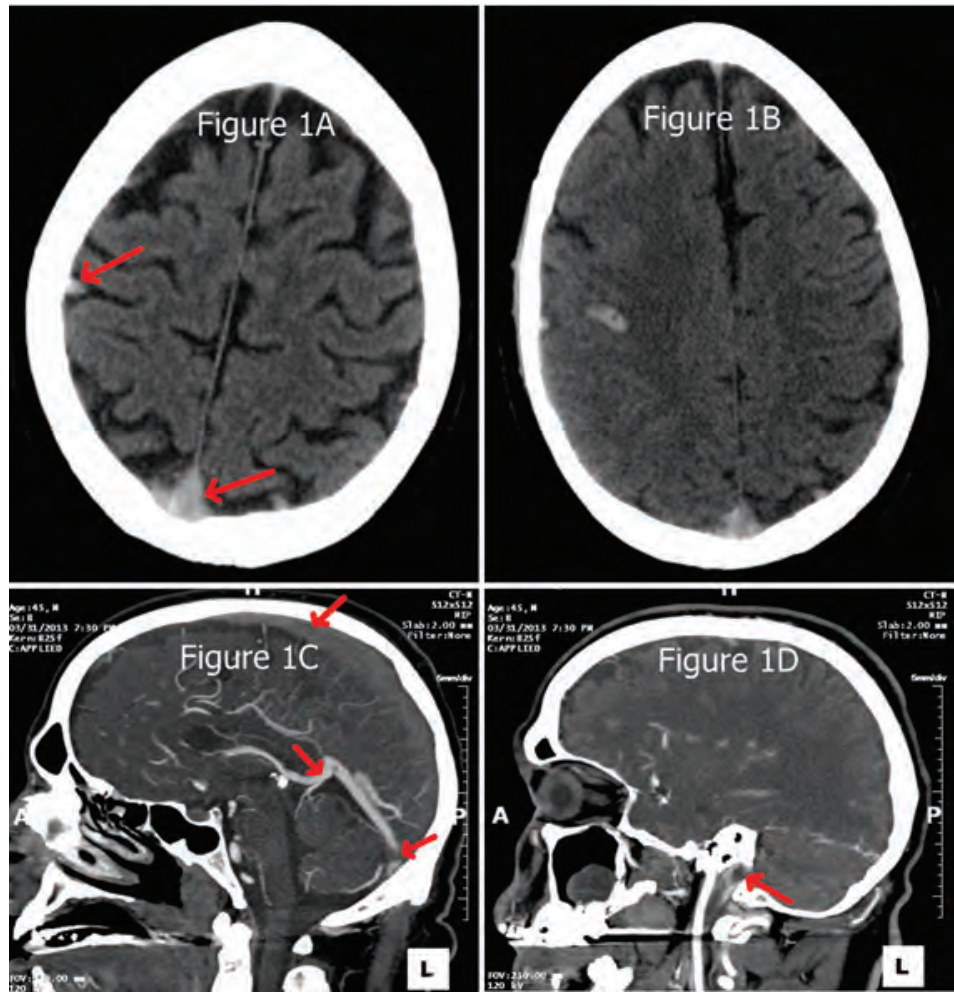

Figure 1A - Non contrast CT - Vein of Trolard (VOT) (left arrow) \& Delta ( $\Delta$ ) Signs (bottom middle arrow); Figure $1 \mathrm{~B}$ - Non Contrast CT - VOT and $\Delta$ signs, as well as right subcortical parenchymal hemorrhage from venous hypertension; Figure 1C - Sagittal CT venogram - Sagittal sinus thrombosis (top arrow), patent vein of Galen (middle arrow) and thrombus at the confluence of the transverse and straight Sinuses (bottom right arrow); Figure 1D - Sagittal CT venogram - Thrombus at the top of the jugular vein at the jugular foramen (arrow).

Authors' contributions: Conception and design: WDF, TR; Acquisition, analysis, and interpretation of data: WDF, TR; Drafting the article: WDF, TR; Revising it critically for important intellectual content: WDF, TR.

Conflict of interest: The authors declare that they have no conflict of interest.

Corresponding author:

William D. Freeman

Department of Neurology

Mayo Clinic

4500 San Pablo Rd.

Cannaday 2 East Building

Jacksonville, FL 32224

freeman.william1@mayo.edu

Tel.: + 904953 7103; Fax.: + 9049530760

Received: 28 June 2013; Accepted: 22 January 2014

Copyright (@) 2014 by Academy of Sciences and Arts of Bosnia and Herzegovina. E-mail for permission to publish: amabih@anubih.ba

\section{References}

1. Chang R, Friedman DP. Isolated cortical venous thrombosis presenting as subarachnoid hemorrhage: a report of three cases. Am J Neuroradiol. 2004;25(10):1676-9.

2. International Team for the Revision of the International Criteria for Behçet's Disease (ITRICBD): Davatchi F, Assaad-Khalil S, Calamia KT, et al. (2013) The International Criteria for Behçet's Disease (ICBD): a collaborative study of 27 countries on the sensitivity and specificity of the new criteria. J Eur Acad Dermatol Venereol. doi: 10.1111/ jdv.12107.

3. Saposnik G, Barinagarrementeria F, Brown RD Jr, Bushnell CD, Cucchiara B, Cushman M, et al. Diagnosis and management of cerebral venous thrombosis: a statement for healthcare professionals from the American Heart Association/American Stroke Association. Stroke. 2011;42:1158. 